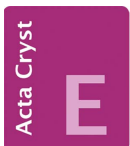

CRYSTALLOGRAPHIC COMMUNICATIONS

ISSN 2056-9890

Received 18 October 2019

Accepted 21 October 2019

Edited by $\mathrm{H}$. Stoeckli-Evans, University of Neuchâtel, Switzerland

Keywords: crystal structure; supramolecular architecture; $\mathrm{C}-\mathrm{H} \ldots \mathrm{Cl}$ hydrogen bonds; $\pi-\pi$ interactions; Hirshfeld surface analysis.

CCDC reference: 1449647

Supporting information: this article has supporting information at journals.iucr.org/e
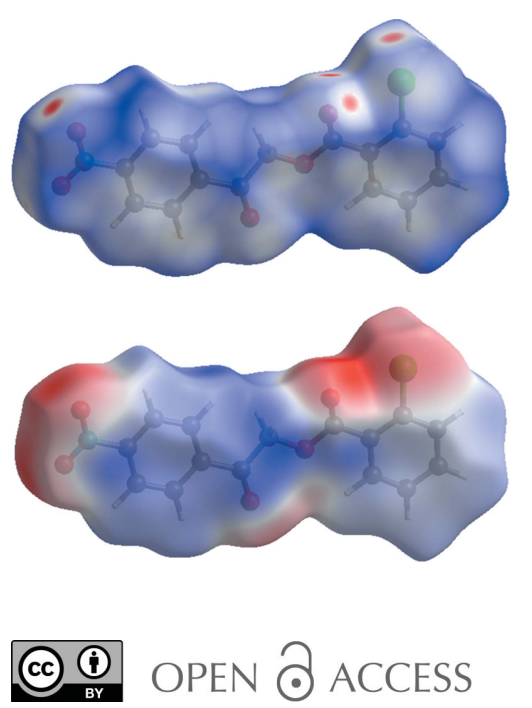

\section{Crystal structure and Hirshfeld surface analysis of 2-(4-nitrophenyl)-2-oxoethyl 2-chlorobenzoate}

\author{
S. N. Sheshadri, ${ }^{a}$ C. S. Chidan Kumar, ${ }^{b}$ S. Naveen, ${ }^{c *}$ M. K. Veeraiah, ${ }^{\text {d }}$ Kakarla \\ Raghava Reddy ${ }^{\mathrm{e}}$ and Ismail Warad ${ }^{\mathrm{f}}$ *
}

a'Department of Chemistry, GSSS Institute of Engineering and Technology for Women, Mysuru 570016, Karnataka, India,
${ }^{\mathbf{b}}$ Department of Engineering Chemistry, Vidya Vikas Institute of, Engineering \& Technology, Visvesvaraya Technological
University, Alanahally, Mysuru 570028 , Karnataka, India, ' ${ }^{\mathbf{C}}$ Department of Physics, School of Engineering and
Technology, Jain University, Bangalore 562112 , India, ${ }^{\mathbf{d}}$ Department of Chemistry, Sri Siddhartha Institute of Technology,
Tumkur 572105 , Karnataka, India, ${ }^{\mathbf{e}}$ School of Chemical \& Biomolecular Engineering, The University of Sydney, Sydney,
NSW, Australia, and 'Department of Chemistry, Science College, An-Najah National University, PO Box 7, Nablus, West
Bank, Palestinian Territories. *Correspondence e-mail: s.naveen@jainuniversity.ac.in, khalil.i@najah.edu

The title compound, $\mathrm{C}_{15} \mathrm{H}_{10} \mathrm{ClNO}_{5}$, is relatively planar with the two aromatic rings being inclined to each other by $3.56(11)^{\circ}$. The central $-\mathrm{C}(=\mathrm{O})-\mathrm{C}-\mathrm{O}-$ $\mathrm{C}(=\mathrm{O})-$ bridge is slightly twisted, with a $\mathrm{C}-\mathrm{C}-\mathrm{O}-\mathrm{C}$ torsion angle of $164.95(16)^{\circ}$. In the crystal, molecules are linked by $\mathrm{C}-\mathrm{H} \cdots \mathrm{O}$ and $\mathrm{C}-\mathrm{H} \cdots \mathrm{Cl}$ hydrogen bonds, forming layers parallel to the (101) plane. The layers are linked by a further $\mathrm{C}-\mathrm{H} \cdots \mathrm{O}$ hydrogen bond, forming a three-dimensional supramolecular structure. There are a number of offset $\pi-\pi$ interactions present between the layers [intercentroid distances vary from $3.8264(15)$ to $3.9775(14) \AA]$. Hirshfeld surface analyses, the $d_{\text {norm }}$ surfaces, electrostatic potential and two-dimensional fingerprint plots were examined to verify the contributions of the different intermolecular contacts within the supramolecular structure. The shape-index surface shows that two sides of the molecule are involved in the same contacts with neighbouring molecules, and the curvedness plot shows flat surface patches that are characteristic of planar stacking.

\section{Chemical context}

Due to their numerous applications in various fields of chemistry, phenacyl benzoates are of great importance (Rather \& Reid, 1919; Literák et al., 2006; Sheehan \& Umezawa, 1973; Huang et al., 1996; Gandhi et al., 1995; Zhang et al., 2009). In continuation of our work on such molecules (Kumar et al., 2014; Chidan Kumar et al., 2014), we report herein on the crystal and molecular structures of 2-(4-nitrophenyl)-2-oxoethyl chlorobenzoate (I). Its crystal and molecular structures are compared with those of 2-(4nitrophenyl)-2-oxoethyl benzoate (II) (Sheshadri et al., 2019), published by us recently, and further details of uses and applications of such molecules are described therein.

\section{Structural commentary}

The molecular structure of the title compound, I, is shown in Fig. 1. The compound is composed of two aromatic rings ( $\mathrm{C} 1-$ $\mathrm{C} 6$ and $\mathrm{C} 10-\mathrm{C} 15)$ linked by the $-\mathrm{C} 7(=\mathrm{O} 2)-\mathrm{C} 8-\mathrm{O} 1-$ $\mathrm{C} 9(=\mathrm{O} 3)$ - bridge. The bond lengths and angles in I are normal and similar to those reported for compound II. The two benzene rings are inclined to each other by $3.56(11)^{\circ}$, indicating that they are almost coplanar, as in the structure of II. The nitro group (N1/O4/O5) lies almost in the plane of the benzene ring (C1-C6), with a dihedral angle between the two 
Table 1

Hydrogen-bond geometry $\left(\AA,^{\circ}\right)$.

\begin{tabular}{lllll}
\hline$D-\mathrm{H} \cdots A$ & $D-\mathrm{H}$ & $\mathrm{H} \cdots A$ & $D \cdots A$ & $D-\mathrm{H} \cdots A$ \\
\hline $\mathrm{C} 2-\mathrm{H} 2 \cdots \mathrm{O}^{\mathrm{i}}$ & 0.93 & 2.54 & $3.258(2)$ & 135 \\
$\mathrm{C} 8-\mathrm{H} 8 A \cdots 3^{\mathrm{ii}}$ & 0.97 & 2.59 & $3.553(3)$ & 171 \\
$\mathrm{C} 13-\mathrm{H} 13 \cdots \mathrm{Cl}^{\mathrm{iii}}$ & 0.93 & 2.82 & $3.670(3)$ & 153 \\
$\mathrm{C} 14-\mathrm{H} 14 \cdots 4^{\mathrm{i}}$ & 0.93 & 2.50 & $3.211(4)$ & 134 \\
\hline
\end{tabular}

Symmetry codes: (i) $-x+1, y+\frac{1}{2},-z+\frac{1}{2}$; (ii) $x,-y+\frac{1}{2}, z-\frac{1}{2}$; (iii) $-x, y+\frac{1}{2},-z+\frac{3}{2}$.

planes of $5.4(4)^{\circ}$; the torsion angles $\mathrm{C} 4-\mathrm{C} 3-\mathrm{N} 1-\mathrm{O} 4$ and $\mathrm{C} 2-\mathrm{C} 3-\mathrm{N} 1-\mathrm{O} 5$ are $-5.4(3)$ and $-5.1(4)^{\circ}$, respectively. Atom $\mathrm{Cl} 1$ is displaced by 0.0749 (8) $\AA$ from the plane of benzene ring $\mathrm{C} 10-\mathrm{C} 15$.<smiles>[X]c1ccccc1C(=O)OCC(=O)c1ccc([N+](=O)[O-])cc1</smiles>

The overall molecular conformation of $\mathrm{I}$ is characterized by three torsion angles, viz. $\tau_{1}(\mathrm{C} 11-\mathrm{C} 10-\mathrm{C} 9-\mathrm{O} 3), \tau_{2}(\mathrm{C} 7-$ $\mathrm{C} 8-\mathrm{O} 1-\mathrm{C} 9)$ and $\tau_{3}(\mathrm{O} 2-\mathrm{C} 7-\mathrm{C} 6-\mathrm{C} 1)$. Torsion angle $\tau_{1}$ at $-12.5(3)^{\circ}$ signifies a certain noncoplanarity between the benzene ring $(\mathrm{C} 10-\mathrm{C} 15)$ and the adjacent carbonyl group $(\mathrm{C} 9=\mathrm{O} 3)$ as a result of steric repulsion between the substituent $\mathrm{Cl} 1$ and the adjacent carbonyl group $\mathrm{C} 9=\mathrm{O} 3$. This is also reflected in the torsion angle $\tau_{2}$ of $-164.95(16)^{\circ}$, between the two carbonyl groups, $\mathrm{C} 7=\mathrm{O} 2$ and $\mathrm{C} 9=\mathrm{O} 3$, which have a antiperiplanar conformation. Torsion angle $\tau_{3}$, involving the benzene ring (C1-C6) and the adjacent carbonyl group $(\mathrm{C} 7=\mathrm{O} 2)$, is $-3.6(3)^{\circ}$ and indicates a -synperiplanar conformation. A comparison of the torsion angles in I and II, indicates that the insertion of the $\mathrm{Cl}$ atom in $\mathrm{I}$ has the most significant influence on torsion $\tau_{2}$, which is $-164.95(16)^{\circ}$ in I compared to $174.08(9)^{\circ}$ in II. Torsion angles $\tau_{1}$ of $-12.5(3)^{\circ}$ and $\tau_{3}$ of $-3.6(3)^{\circ}$ are slightly larger than the values observed in II, viz. $9.60(16)$ and $1.88(15)^{\circ}$, respectively. Hence,

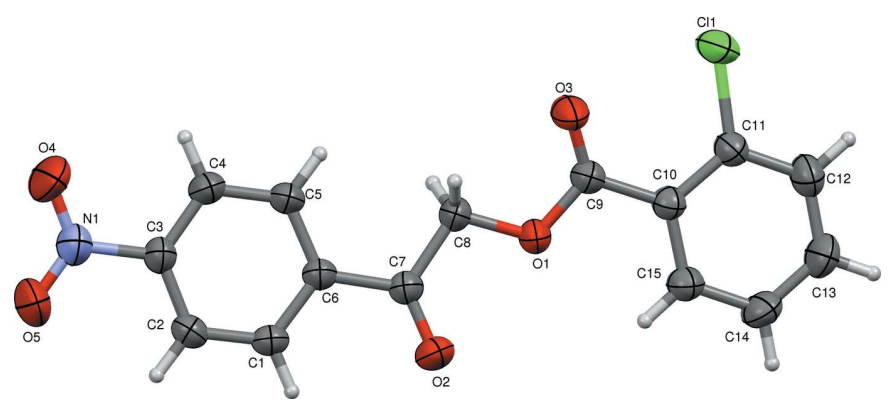

Figure 1

The molecular structure of compound I, with the atom labelling. Displacement ellipsoids are drawn at the $50 \%$ probability level.

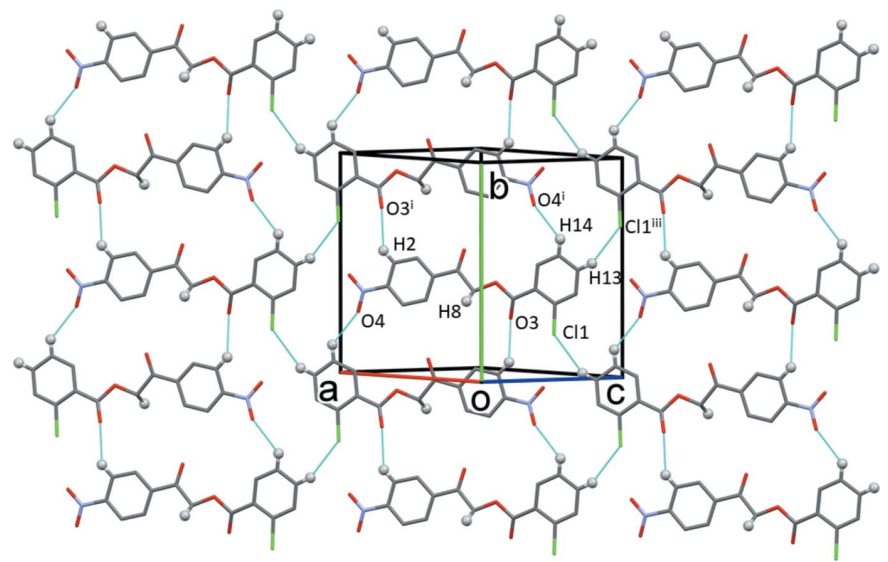

Figure 2

A view normal to the (101) plane of the crystal packing of compound I. The hydrogen bonds are shown as dashed lines (Table 1; symmetry codes as in Table 1), and, for clarity, only the $\mathrm{H}$ atoms involved in hydrogen bonding have been included.

compound I has a less planar conformation than unsubstituted compound II.

\section{Supramolecular features}

The crystal structure of the title compound, is stabilized by intermolecular hydrogen bonds of the types $\mathrm{C}-\mathrm{H} \cdots \mathrm{O}$ and $\mathrm{C}-\mathrm{H} \cdots \mathrm{Cl}$ (Table 1). Molecules are linked by the $\mathrm{C} 2-$ $\mathrm{H} 2 \cdots \mathrm{O} 3^{\mathrm{i}}, \mathrm{C} 14-\mathrm{H} 14 \cdots \mathrm{O} 4^{\mathrm{i}}$ and $\mathrm{C} 13-\mathrm{H} 13 \cdots \mathrm{Cl} 1^{\mathrm{iii}}$ hydrogen bonds to form layers lying parallel to the (101) plane; see Fig. 2 and Table 1 . The layers are linked by $\mathrm{C} 8-\mathrm{H} 8 A \cdots \mathrm{O} 3^{\mathrm{ii}}$ hydrogen bonds and offset $\pi-\pi$ interactions (see Table 2), forming a supramolecular three-dimensional structure (Fig. 3).

\section{Hirshfeld surface analysis and two-dimensional fingerprint plots}

The Hirshfeld surface analysis (Spackman \& Jayatilaka, 2009) and the associated two-dimensional fingerprint plots (McKinnon et al., 2007) were performed with Crystal-

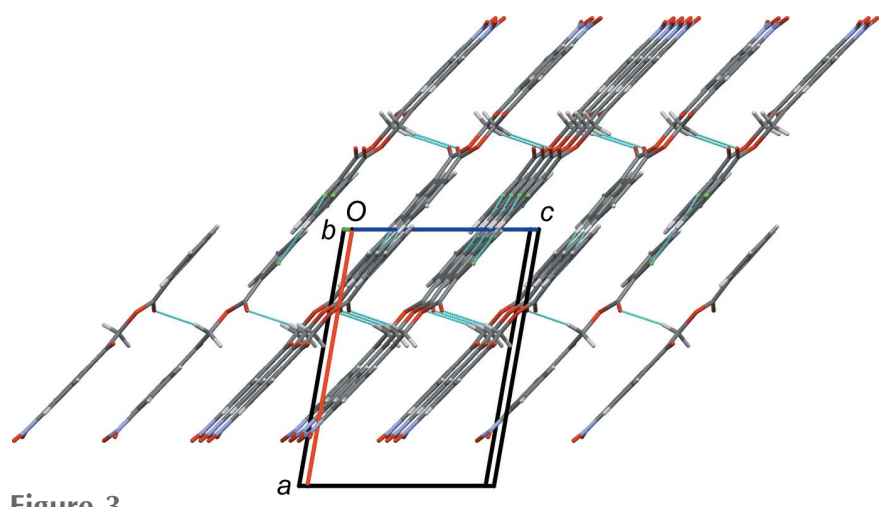

Figure 3

The crystal packing of compound I, viewed along the $b$ axis, showing the layered stacking. For clarity, only the $\mathrm{H}$ atoms involved in hydrogen bonding have been included, and the hydrogen bonds are shown as dashed lines (Table 1). 
Table 2

$\pi-\pi$ contacts $\left(\AA,^{\circ}\right)$ in the crystal of compound $\mathrm{I}$.

$\mathrm{Cg} 1$ and $\mathrm{Cg} 2$ are the centroids of the $\mathrm{C} 1-\mathrm{C} 6$ and $\mathrm{C} 10-\mathrm{C} 15$ rings, respectively.

\begin{tabular}{lllllllll}
\hline$C g(I)$ & $C g(J)$ & $C g(I) \cdots C g(J)(\AA)$ & $\alpha\left(^{\circ}\right)$ & $\beta\left(^{\circ}\right)$ & $\gamma\left(^{\circ}\right)$ & $C g I+P e r p(\AA)$ & $C g J \_P e r p(\AA)$ & Offset $(\AA)$ \\
\hline$C g 1$ & $C g 1^{\text {iv }}$ & $3.9775(14)$ & $0.02(10)$ & 31.8 & 31.8 & $3.3791(9)$ & $3.3791(9)$ & 2.098 \\
$C g 1$ & $C g 2^{\mathrm{v}}$ & $3.8801(14)$ & $3.56(11)$ & 30.1 & 29.1 & $3.3895(9)$ & $3.3559(10)$ & 1.948 \\
$C g 2$ & $C g 2^{\mathrm{vi}}$ & $3.8264(15)$ & $0.00(11)$ & 24.8 & 24.8 & $3.4722(10)$ & $3.4722(10)$ & 1.608 \\
\hline
\end{tabular}

Symmetry codes: (iv) $-x+1,-y+1,-z$; (v) $-x+1,-y+1,-z+1 ;($ vi) $-x,-y+1,-z+1$.

Explorer17 (Turner et al., 2017). Hirshfeld surface analysis enables the visualization of intermolecular interactions by different colours and colour intensity, representing short or long contacts and indicating the relative strength of the interactions. Fig. 4(a) shows the Hirshfeld surface mapped over $d_{\text {norm }}(-0.154$ to 1.305$)$ and for Fig. 4(b) the electrostatic potential. The Hirshfeld surface illustrated in Fig. 4(a) reflects the involvement of different atoms with the intermolecular interactions through the appearance of blue and red patches, which correspond to the regions of positive and negative electrostatic potential shown in Fig. 4(b). The shape-index surface (Fig. 5a) clearly shows that the two sides of the molecule are involved in contacts with neighbouring molecules and the curvedness plot (Fig. $5 b$ ) shows flat surface patches characteristic of planar stacking.

The overall two-dimensional fingerprint plot for the title compound and those delineated into $\mathrm{O} \cdots \mathrm{H} / \mathrm{H} \cdots \mathrm{O}, \mathrm{H} \cdots \mathrm{H}$, $\mathrm{C} \cdots \mathrm{H} / \mathrm{H} \cdots \mathrm{C}$ and $\mathrm{Cl} \cdots \mathrm{H} / \mathrm{H} \cdots \mathrm{Cl}$ contacts are illustrated in Fig. 6. The percentage contributions from the different interatomic contacts to the Hirshfeld surfaces are as follows: $\mathrm{O} \cdots \mathrm{H}$ $(34.8 \%), \mathrm{H} \cdots \mathrm{H}(18.8 \%), \mathrm{C} \cdots \mathrm{H}(14.7 \%)$ and $\mathrm{Cl} \cdots \mathrm{H}(10.1 \%)$,
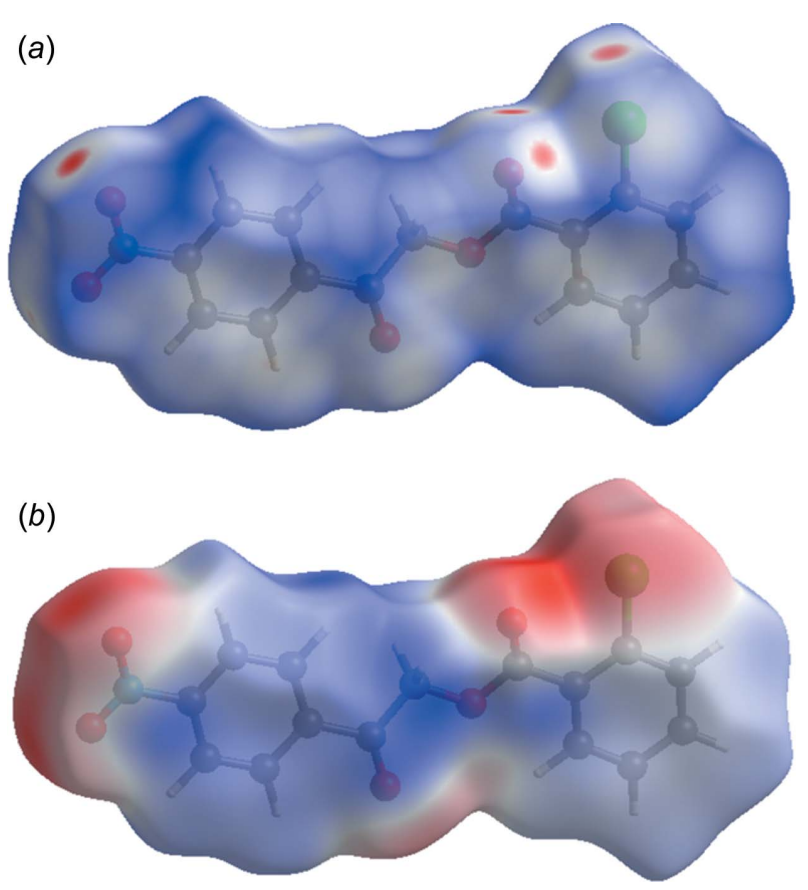

Figure 4

A view of the Hirshfeld surface of compound I, mapped over $d_{\text {norm. }}$. shown in the two-dimensional fingerprint plots, respectively, in Fig. 6. The percentage contributions for other intermolecular contacts are less than $5 \%$ in the Hirshfeld surface mapping.

\section{Database survey}

A search of the Cambridge Structural Database (CSD, Version 5.40, last update May 2019; Groom et al., 2016) using 2-oxo-2-phenylethyl benzoate as the main skeleton revealed the presence of 62 structures with different substituents on the terminal phenyl rings. In these structures, the two aromatic rings are inclined to each other by dihedral angles varying from $c a 0$ to $90^{\circ}$. There were seven structures with a nitro substituent on one of the aromatic rings. However, there is only one compound with the same skeleton as the title compound, i.e. 2-(biphenyl-4-yl)-2-oxoethyl 4-nitrobenzoate (CSD refcode CISSAB; Kwong et al., 2017). Here the two aromatic rings are inclined to each other by $c a 70.96^{\circ}$, compared to only $3.56(11)^{\circ}$ in the title compound. In the crystal structure of the recently published compound 2-(4-
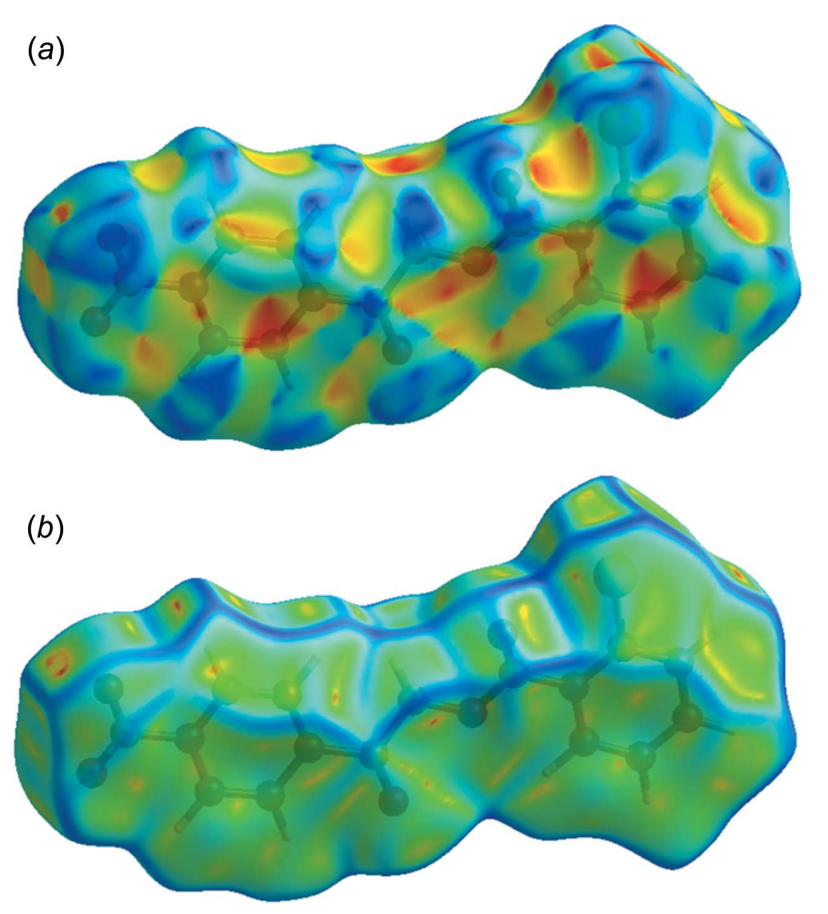

Figure 5

Hirshfeld surface of compound I, mapped over (a) the shape-index and (b) the curvedness. 
nitrophenyl)-2-oxoethyl benzoate (II) (Sheshadri et al., 2019), this dihedral angle is $3.09(5)^{\circ}$.

\section{Synthesis and crystallization}

The title compound, was synthesized as per the procedure reported earlier by Kumar et al. (2014). A mixture of 2-bromo1-(4-nitrophenyl)ethanone $\quad(0.2 \mathrm{~g}, \quad 0.5 \mathrm{mmol}), \quad$ potassium carbonate $(0.087 \mathrm{~g}, 0.63 \mathrm{mmol})$ and 2-chlorobenzoic acid $(0.156 \mathrm{~g}, 0.65 \mathrm{mmol})$ in dimethylformamide $(5 \mathrm{ml})$ was stirred at room temperature for $2 \mathrm{~h}$. After completion of the reaction, the reaction mixture was poured into ice-cold water. The solid product obtained was filtered, washed with water and recrystallized from ethanol to give colourless block-like crystals (m.p. 386-390 K).

\section{Refinement}

Crystal data, data collection and structure refinement details are summarized in Table 3. C-bound $\mathrm{H}$ atoms were positioned geometrically $(\mathrm{C}-\mathrm{H}=0.93-0.97 \AA)$ and refined using a riding model, with $U_{\text {iso }}(\mathrm{H})=1.2 U_{\mathrm{eq}}(\mathrm{C})$.

\section{Acknowledgements}

CSCK extends his appreciation to Vidya Vikas Research \& Development Centre for the facilities and encouragement. NS thanks Jain University for sanctioning research grants under minor project.

\section{References}

Bruker (2012). APEX2, SAINT and SADABS. Bruker AXS Inc., Madison, Wisconsin, USA.
Table 3

Experimental details.

\begin{tabular}{|c|c|}
\hline \multicolumn{2}{|l|}{ Crystal data } \\
\hline Chemical formula & $\mathrm{C}_{15} \mathrm{H}_{10} \mathrm{ClNO}_{5}$ \\
\hline$M_{\mathrm{r}}$ & 319.69 \\
\hline Crystal system, space group & Monoclinic, $P 2_{1} / c$ \\
\hline Temperature $(\mathrm{K})$ & 294 \\
\hline$a, b, c(\AA)$ & $\begin{array}{l}12.6646(18), 12.4099(18) \\
\quad 9.0902(13)\end{array}$ \\
\hline$\beta\left(^{\circ}\right)$ & $99.947(2)$ \\
\hline$V\left(\AA^{3}\right)$ & $1407.2(3)$ \\
\hline$Z$ & 4 \\
\hline Radiation type & Mo $K \alpha$ \\
\hline$\mu\left(\mathrm{mm}^{-1}\right)$ & 0.30 \\
\hline Crystal size $(\mathrm{mm})$ & $0.55 \times 0.26 \times 0.19$ \\
\hline \multicolumn{2}{|l|}{ Data collection } \\
\hline Diffractometer & $\begin{array}{l}\text { Bruker APEXII DUO CCD area- } \\
\text { detector }\end{array}$ \\
\hline Absorption correction & $\begin{array}{l}\text { Multi-scan (SADABS; Bruker, } \\
\text { 2012) }\end{array}$ \\
\hline$T_{\min }, T_{\max }$ & $0.796,0.946$ \\
\hline $\begin{array}{l}\text { No. of measured, independent and } \\
\text { observed }[I>2 \sigma(I)] \text { reflections }\end{array}$ & $36449,4122,2586$ \\
\hline$R_{\text {int }}$ & 0.058 \\
\hline$(\sin \theta / \lambda)_{\max }\left(\AA^{-1}\right)$ & 0.705 \\
\hline \multicolumn{2}{|l|}{ Refinement } \\
\hline$R\left[F^{2}>2 \sigma\left(F^{2}\right)\right], w R\left(F^{2}\right), S$ & $0.054,0.141,1.06$ \\
\hline No. of reflections & 4122 \\
\hline No. of parameters & 199 \\
\hline $\mathrm{H}$-atom treatment & $\mathrm{H}$-atom parameters constrained \\
\hline$\Delta \rho_{\max }, \Delta \rho_{\min }\left(\mathrm{e} \AA^{-3}\right)$ & $0.26,-0.44$ \\
\hline
\end{tabular}

Computer programs: APEX2 (Bruker, 2012), SAINT (Bruker, 2012), SHELXS97 (Sheldrick, 2008), Mercury (Macrae et al., 2008), SHELXL97 (Sheldrick, 2008), PLATON (Spek, 2009) and publCIF (Westrip, 2010).

Chidan Kumar, C. S., Fun, H.-K., Tursun, M., Ooi, C. W., Chandraju, S., Quah, C. K. \& Parlak, C. (2014). Spectrochim. Acta A Mol. Biomol. Spectrosc. 124, 595-602.

Figure 6

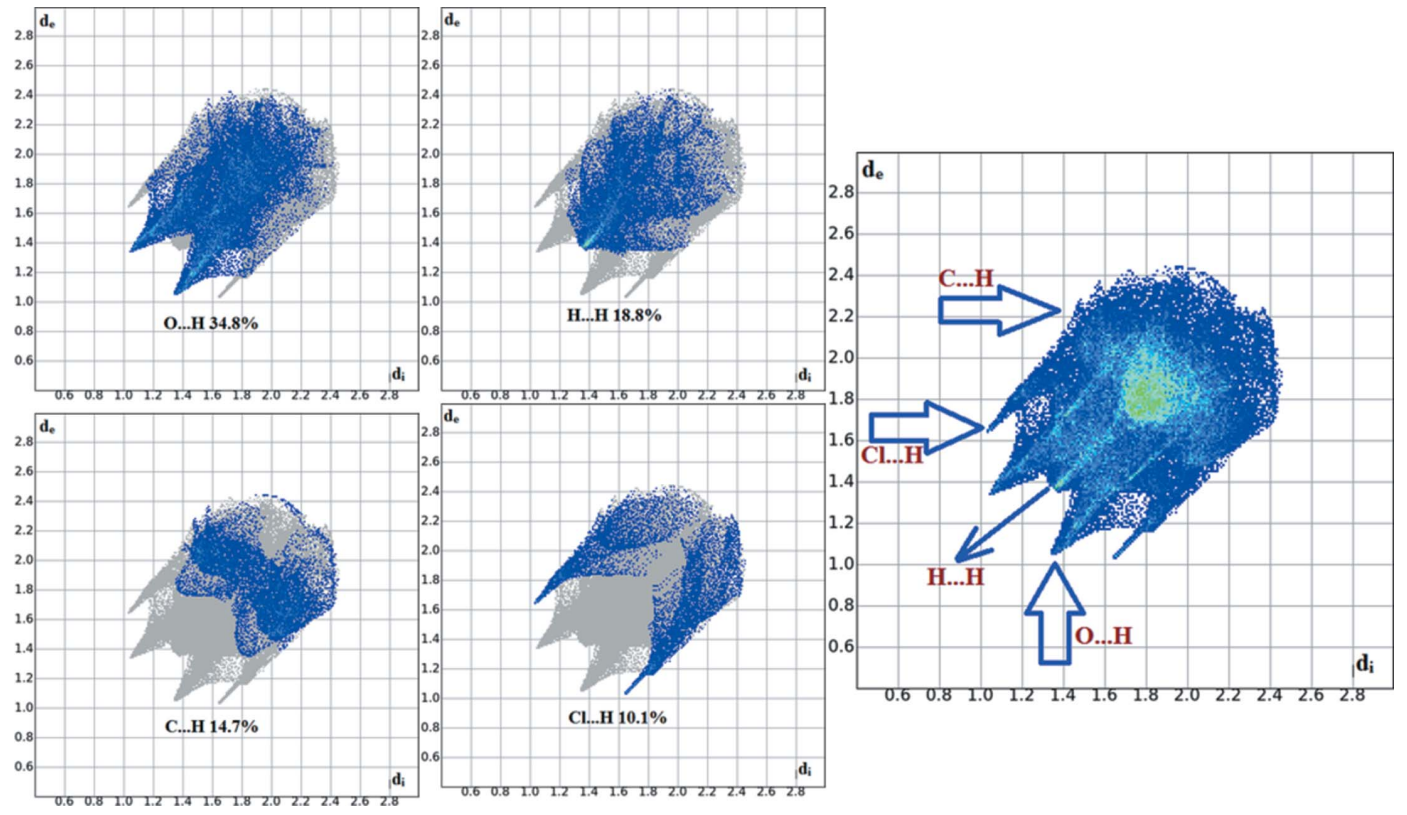

The two-dimensional fingerprint plots of compound I, showing the percentage contributions of all contacts and of individual atom-atom contacts. 
Gandhi, S. S., Bell, K. L. \& Gibson, M. S. (1995). Tetrahedron, 51, 13301-13308.

Groom, C. R., Bruno, I. J., Lightfoot, M. P. \& Ward, S. C. (2016). Acta Cryst. B72, 171-179.

Huang, W., Pei, J., Chen, B., Pei, W. \& Ye, X. (1996). Tetrahedron, 52, 10131-10136.

Kumar, C. S. C., Chia, T. S., Ooi, C. W., Quah, C. K., Chandraju, S. \& Fun, H. K. (2014). Z. Kristallogr. Cryst. Mater. 229, 328-342.

Kwong, H. C., Chidan Kumar, C. S., Mah, S. H., Chia, T. S., Quah, C. K., Loh, Z. H., Chandraju, S. \& Lim, G. K. (2017). PLoS One, 12, e0170117.

Literák, J., Dostálová, A. \& Klán, P. (2006). J. Org. Chem. 71, 713723.

Macrae, C. F., Bruno, I. J., Chisholm, J. A., Edgington, P. R., McCabe, P., Pidcock, E., Rodriguez-Monge, L., Taylor, R., van de Streek, J. \& Wood, P. A. (2008). J. Appl. Cryst. 41, 466-470.
McKinnon, J. J., Jayatilaka, D. \& Spackman, M. A. (2007). Chem. Commun. pp. 3814.

Rather, J. B. \& Reid, E. (1919). J. Am. Chem. Soc. 41, 75-83.

Sheehan, J. C. \& Umezawa, K. (1973). J. Org. Chem. 38, 3771-3774.

Sheldrick, G. M. (2008). Acta Cryst. A64, 112-122.

Sheshadri, S. N., Chidan Kumar, C. S., Naveen, S., Veeraiah, M. K., Raghava Reddy, K. \& Warad, I. (2019). Acta Cryst. E75, 1719-1723. Spackman, M. A. \& Jayatilaka, D. (2009). CrystEngComm, 11, 19-32. Spek, A. L. (2009). Acta Cryst. D65, 148-155.

Turner, M. J., McKinnon, J. J., Wolff, S. K., Grimwood, D. J., Spackman, P. R., Jayatilaka, D. \& Spackman, M. A. (2017). CrystalExplorer17. University of Western Australia. http://hirshfeldsurface.net.

Westrip, S. P. (2010). J. Appl. Cryst. 43, 920-925.

Zhang, L., Shen, Y., Zhu, H. J., Wang, F., Leng, Y. \& Liu, J. K. (2009). J. Antibiot. 62, 239-242. 


\section{supporting information}

Acta Cryst. (2019). E75, 1792-1796 [https://doi.org/10.1107/S2056989019014336]

Crystal structure and Hirshfeld surface analysis of 2-(4-nitrophenyl)-2-oxoethyl 2-chlorobenzoate

S. N. Sheshadri, C. S. Chidan Kumar, S. Naveen, M. K. Veeraiah, Kakarla Raghava Reddy and Ismail Warad

Computing details

Data collection: APEX2 (Bruker, 2012); cell refinement: SAINT (Bruker, 2012); data reduction: SAINT (Bruker, 2012); program(s) used to solve structure: SHELXS97 (Sheldrick, 2008); program(s) used to refine structure: SHELXL97 (Sheldrick, 2008); molecular graphics: Mercury (Macrae et al., 2008); software used to prepare material for publication: SHELXL97 (Sheldrick, 2008), PLATON (Spek, 2009) and publCIF (Westrip, 2010).

2-(4-Nitrophenyl)-2-oxoethyl 2-chlorobenzoate

Crystal data

$\mathrm{C}_{15} \mathrm{H}_{10} \mathrm{ClNO}_{5}$

$M_{r}=319.69$

Monoclinic, $P 2_{1} / c$

Hall symbol: -P $2 \mathrm{ybc}$

$a=12.6646(18) \AA$

$b=12.4099(18) \AA$

$c=9.0902(13) \AA$

$\beta=99.947(2)^{\circ}$

$V=1407.2(3) \AA^{3}$

$Z=4$

\section{Data collection}

Bruker APEXII DUO CCD area-detector diffractometer

Radiation source: Rotating Anode

Graphite monochromator

Detector resolution: 18.4 pixels $\mathrm{mm}^{-1}$

$\varphi$ and $\omega$ scans

Absorption correction: multi-scan

(SADABS; Bruker, 2012)

$T_{\min }=0.796, T_{\max }=0.946$

Refinement

Refinement on $F^{2}$

Least-squares matrix: full

$R\left[F^{2}>2 \sigma\left(F^{2}\right)\right]=0.054$

$w R\left(F^{2}\right)=0.141$

$S=1.06$

4122 reflections
$F(000)=656$

$D_{\mathrm{x}}=1.509 \mathrm{Mg} \mathrm{m}^{-3}$

Mo $K \alpha$ radiation, $\lambda=0.71073 \AA$

Cell parameters from 6264 reflections

$\theta=2.3-27.5^{\circ}$

$\mu=0.30 \mathrm{~mm}^{-1}$

$T=294 \mathrm{~K}$

Block, colourless

$0.55 \times 0.26 \times 0.19 \mathrm{~mm}$

36449 measured reflections

4122 independent reflections

2586 reflections with $I>2 \sigma(I)$

$R_{\text {int }}=0.058$

$\theta_{\max }=30.1^{\circ}, \theta_{\min }=1.6^{\circ}$

$h=-17 \rightarrow 17$

$k=-17 \rightarrow 17$

$l=-12 \rightarrow 12$

199 parameters

0 restraints

Hydrogen site location: inferred from neighbouring sites

$\mathrm{H}$-atom parameters constrained 
$\mathrm{W}=1 /\left[\Sigma^{2}\left(F \mathrm{O}^{2}\right)+(0.0456 P)^{2}+0.8032 P\right]$
WHERE $P=\left(F \mathrm{O}^{2}+2 F \mathrm{C}^{2}\right) / 3$
$(\Delta / \sigma)_{\max }<0.001$

\section{Special details}

Geometry. Bond distances, angles etc. have been calculated using the rounded fractional coordinates. All su's are estimated from the variances of the (full) variance-covariance matrix. The cell esds are taken into account in the estimation of distances, angles and torsion angles

Refinement. Refinement on $\mathrm{F}^{2}$ for ALL reflections except those flagged by the user for potential systematic errors. Weighted R-factors $\mathrm{wR}$ and all goodnesses of fit $\mathrm{S}$ are based on $\mathrm{F}^{2}$, conventional R-factors $\mathrm{R}$ are based on $\mathrm{F}$, with $\mathrm{F}$ set to zero for negative $\mathrm{F}^{2}$. The observed criterion of $\mathrm{F}^{2}>2 \operatorname{sigma}\left(\mathrm{F}^{2}\right)$ is used only for calculating -R-factor-obs etc. and is not relevant to the choice of reflections for refinement. R-factors based on $\mathrm{F}^{2}$ are statistically about twice as large as those based on F, and R-factors based on ALL data will be even larger.

Fractional atomic coordinates and isotropic or equivalent isotropic displacement parameters $\left(\AA^{2}\right)$

\begin{tabular}{|c|c|c|c|c|}
\hline & $x$ & $y$ & $z$ & $U_{\text {iso }} * / U_{\text {eq }}$ \\
\hline $\mathrm{Cl1}$ & $0.13276(6)$ & $0.19871(4)$ & $0.64688(9)$ & 0.0704 \\
\hline $\mathrm{O} 1$ & $0.32241(12)$ & $0.43016(11)$ & $0.42430(17)$ & $0.0451(5)$ \\
\hline $\mathrm{O} 2$ & $0.44530(13)$ & $0.57459(11)$ & 0.32308 (19) & $0.0553(6)$ \\
\hline $\mathrm{O} 3$ & $0.31616(15)$ & $0.26717(12)$ & $0.5238(2)$ & $0.0662(7)$ \\
\hline $\mathrm{O} 4$ & $0.8091(3)$ & $0.28826(18)$ & -0.0648 (4) & $0.1349(15)$ \\
\hline O5 & $0.8233(2)$ & $0.45298(17)$ & $-0.1127(3)$ & $0.1031(10)$ \\
\hline N1 & $0.78442(18)$ & $0.38048(17)$ & -0.0532 & $0.0632(8)$ \\
\hline $\mathrm{C} 1$ & $0.60280(16)$ & $0.53537(15)$ & $0.1467(2)$ & $0.0378(6)$ \\
\hline $\mathrm{C} 2$ & $0.68013(17)$ & $0.51241(15)$ & $0.0615(2)$ & $0.0407(6)$ \\
\hline $\mathrm{C} 3$ & $0.70239(17)$ & $0.40590(16)$ & $0.0379(2)$ & $0.0432(7)$ \\
\hline $\mathrm{C} 4$ & 0.65143 (19) & $0.32166(16)$ & 0.0961 & $0.0493(7)$ \\
\hline $\mathrm{C} 5$ & $0.57408(18)$ & $0.34589(15)$ & $0.1802(3)$ & $0.0458(7)$ \\
\hline C6 & $0.54870(15)$ & $0.45265(14)$ & $0.2066(2)$ & $0.0356(6)$ \\
\hline $\mathrm{C} 7$ & $0.46407(16)$ & $0.48220(15)$ & $0.2957(2)$ & $0.0367(6)$ \\
\hline $\mathrm{C} 8$ & $0.40417(18)$ & $0.38936(16)$ & $0.3492(2)$ & $0.0429(6)$ \\
\hline $\mathrm{C} 9$ & $0.28099(17)$ & $0.35666(15)$ & $0.5069(2)$ & $0.0394(6)$ \\
\hline $\mathrm{C} 10$ & $0.19286(15)$ & $0.40267(15)$ & $0.5758(2)$ & $0.0365(6)$ \\
\hline $\mathrm{C} 11$ & $0.12673(17)$ & $0.33809(16)$ & $0.6478(2)$ & $0.0414(6)$ \\
\hline $\mathrm{C} 12$ & $0.05046(19)$ & $0.3840(2)$ & 0.7205 & $0.0548(8)$ \\
\hline C13 & $0.0388(2)$ & $0.4942(2)$ & 0.7222 & $0.0604(9)$ \\
\hline $\mathrm{C} 14$ & 0.10067 (19) & $0.55891(18)$ & $0.6499(3)$ & $0.0544(8)$ \\
\hline $\mathrm{C} 15$ & 0.17715 (17) & $0.51397(16)$ & $0.5769(3)$ & $0.0443(7)$ \\
\hline H1 & 0.58650 & 0.60680 & 0.16450 & $0.0450^{*}$ \\
\hline $\mathrm{H} 2$ & 0.71620 & 0.56730 & 0.02110 & $0.0490 *$ \\
\hline H4 & 0.66890 & 0.25050 & 0.07900 & $0.0590 *$ \\
\hline H5 & 0.53830 & 0.29040 & 0.21980 & $0.0550 *$ \\
\hline H8A & 0.37220 & 0.34550 & 0.26490 & $0.0510^{*}$ \\
\hline H8B & 0.45330 & 0.34460 & 0.41680 & $0.0510^{*}$ \\
\hline H12 & 0.00710 & 0.34040 & 0.76810 & $0.0660^{*}$ \\
\hline H13 & -0.01150 & 0.52480 & 0.77300 & $0.0720^{*}$ \\
\hline H14 & 0.09130 & 0.63320 & 0.64990 & $0.0650^{*}$ \\
\hline H15 & 0.21870 & 0.55860 & 0.52770 & $0.0530 *$ \\
\hline
\end{tabular}


Atomic displacement parameters $\left(\AA^{2}\right)$

\begin{tabular}{lllllll}
\hline & $U^{11}$ & $U^{22}$ & $U^{33}$ & $U^{12}$ & $U^{13}$ & $U^{23}$ \\
\hline C11 & $0.0741(4)$ & $0.0363(3)$ & $0.1119(6)$ & $-0.0032(3)$ & $0.0471(4)$ & $0.0109(3)$ \\
O1 & $0.0529(9)$ & $0.0353(7)$ & $0.0540(9)$ & $0.0031(6)$ & $0.0285(7)$ & $0.0035(6)$ \\
O2 & $0.0648(11)$ & $0.0323(7)$ & $0.0754(11)$ & $-0.0003(7)$ & $0.0311(9)$ & $-0.0077(7)$ \\
O3 & $0.0755(12)$ & $0.0382(8)$ & $0.0983(14)$ & $0.0158(8)$ & $0.0524(11)$ & $0.0190(8)$ \\
O4 & $0.165(3)$ & $0.0587(13)$ & $0.222(3)$ & $0.0172(14)$ & $0.149(3)$ & $-0.0060(16)$ \\
O5 & $0.1241(19)$ & $0.0699(13)$ & $0.144(2)$ & $-0.0058(13)$ & $0.1036(18)$ & $0.0043(13)$ \\
N1 & $0.0670(14)$ & $0.0509(11)$ & $0.0825(16)$ & $-0.0031(10)$ & $0.0433(12)$ & $-0.0073(11)$ \\
C1 & $0.0436(11)$ & $0.0268(8)$ & $0.0437(11)$ & $-0.0021(7)$ & $0.0095(9)$ & $-0.0019(8)$ \\
C2 & $0.0446(11)$ & $0.0342(9)$ & $0.0456(12)$ & $-0.0058(8)$ & $0.0141(9)$ & $0.0031(8)$ \\
C3 & $0.0444(12)$ & $0.0394(10)$ & $0.0504(13)$ & $-0.0019(9)$ & $0.0214(10)$ & $-0.0037(9)$ \\
C4 & $0.0588(14)$ & $0.0296(9)$ & $0.0659(15)$ & $-0.0004(9)$ & $0.0284(12)$ & $-0.0048(9)$ \\
C5 & $0.0554(13)$ & $0.0288(9)$ & $0.0591(14)$ & $-0.0051(9)$ & $0.0268(11)$ & $-0.0009(9)$ \\
C6 & $0.0393(10)$ & $0.0295(8)$ & $0.0392(11)$ & $-0.0031(7)$ & $0.0106(9)$ & $-0.0026(8)$ \\
C7 & $0.0412(11)$ & $0.0320(9)$ & $0.0383(11)$ & $-0.0015(8)$ & $0.0105(9)$ & $-0.0013(8)$ \\
C8 & $0.0517(12)$ & $0.0341(9)$ & $0.0486(12)$ & $0.0005(8)$ & $0.0250(10)$ & $-0.0021(8)$ \\
C9 & $0.0428(11)$ & $0.0322(9)$ & $0.0458(12)$ & $-0.0011(8)$ & $0.0149(9)$ & $0.0010(8)$ \\
C10 & $0.0355(10)$ & $0.0339(9)$ & $0.0410(11)$ & $0.0012(7)$ & $0.0089(9)$ & $0.0032(8)$ \\
C11 & $0.0409(11)$ & $0.0368(9)$ & $0.0491(12)$ & $-0.0001(8)$ & $0.0149(9)$ & $0.0046(9)$ \\
C12 & $0.0500(13)$ & $0.0566(13)$ & $0.0644(16)$ & $0.0006(11)$ & $0.0288(12)$ & $0.0066(12)$ \\
C13 & $0.0540(14)$ & $0.0560(14)$ & $0.0790(18)$ & $0.0104(11)$ & $0.0336(14)$ & $-0.0026(13)$ \\
C14 & $0.0537(14)$ & $0.0369(11)$ & $0.0762(17)$ & $0.0096(9)$ & $0.0214(13)$ & $-0.0022(10)$ \\
C15 & $0.0456(12)$ & $0.0343(10)$ & $0.0553(13)$ & $0.0018(8)$ & $0.0150(10)$ & $0.0030(9)$ \\
& & & & & & \\
& & & & & & \\
& & & & & & \\
& & & & & &
\end{tabular}

Geometric parameters $\left(\AA,{ }^{\circ}\right)$

\begin{tabular}{llll}
\hline $\mathrm{C} 11-\mathrm{C} 11$ & $1.732(2)$ & $\mathrm{C} 10-\mathrm{C} 11$ & $1.401(3)$ \\
$\mathrm{O} 1-\mathrm{C} 8$ & $1.428(3)$ & $\mathrm{C} 10-\mathrm{C} 15$ & $1.396(3)$ \\
$\mathrm{O} 1-\mathrm{C} 9$ & $1.344(2)$ & $\mathrm{C} 11-\mathrm{C} 12$ & $1.384(3)$ \\
$\mathrm{O} 2-\mathrm{C} 7$ & $1.206(2)$ & $\mathrm{C} 12-\mathrm{C} 13$ & $1.376(4)$ \\
$\mathrm{O} 3-\mathrm{C} 9$ & $1.197(2)$ & $\mathrm{C} 13-\mathrm{C} 14$ & $1.383(3)$ \\
$\mathrm{O} 4-\mathrm{N} 1$ & $1.196(3)$ & $\mathrm{C} 14-\mathrm{C} 15$ & 0.9300 \\
$\mathrm{O} 5-\mathrm{N} 1$ & $1.199(3)$ & $\mathrm{C} 1-\mathrm{H} 1$ & 0.9300 \\
$\mathrm{~N} 1-\mathrm{C} 3$ & $1.470(3)$ & $\mathrm{C} 2-\mathrm{H} 2$ & 0.9300 \\
$\mathrm{C} 1-\mathrm{C} 2$ & $1.379(3)$ & $\mathrm{C} 4-\mathrm{H} 4$ & 0.9300 \\
$\mathrm{C} 1-\mathrm{C} 6$ & $1.396(3)$ & $\mathrm{C} 5-\mathrm{H} 5$ & 0.9700 \\
$\mathrm{C} 2-\mathrm{C} 3$ & $1.376(3)$ & $\mathrm{C} 8-\mathrm{H} 8 \mathrm{~A}$ & 0.9700 \\
$\mathrm{C} 3-\mathrm{C} 4$ & $1.381(3)$ & $\mathrm{C} 8-\mathrm{H} 8 \mathrm{~B}$ & 0.9300 \\
$\mathrm{C} 4-\mathrm{C} 5$ & $1.376(3)$ & $\mathrm{C} 12-\mathrm{H} 12$ & 0.9300 \\
$\mathrm{C} 5-\mathrm{C} 6$ & $1.394(3)$ & $\mathrm{C} 13-\mathrm{H} 13$ & 0.9300 \\
$\mathrm{C} 6-\mathrm{C} 7$ & $1.496(3)$ & $\mathrm{C} 14-\mathrm{H} 14$ & 0.9300 \\
$\mathrm{C} 7-\mathrm{C} 8$ & $1.506(3)$ & & \\
$\mathrm{C} 9-\mathrm{C} 10$ & $1.485(3)$ & $\mathrm{C} 10-\mathrm{C} 11-\mathrm{C} 12$ & $120.71(19)$ \\
$\mathrm{C} 8-\mathrm{O} 1-\mathrm{C} 15$ & $\mathrm{C} 11-\mathrm{C} 12-\mathrm{C} 13$ & $120.0(2)$ \\
$\mathrm{O} 4-\mathrm{N} 1-\mathrm{O} 5$ & $114.31(15)$ & &
\end{tabular}




\begin{tabular}{|c|c|c|c|}
\hline $\mathrm{O} 4-\mathrm{N} 1-\mathrm{C} 3$ & $118.4(3)$ & $\mathrm{C} 12-\mathrm{C} 13-\mathrm{C} 14$ & $120.5(2)$ \\
\hline $\mathrm{O} 5-\mathrm{N} 1-\mathrm{C} 3$ & $118.6(2)$ & $\mathrm{C} 13-\mathrm{C} 14-\mathrm{C} 15$ & $120.1(2)$ \\
\hline $\mathrm{C} 2-\mathrm{C} 1-\mathrm{C} 6$ & $120.74(17)$ & $\mathrm{C} 10-\mathrm{C} 15-\mathrm{C} 14$ & $121.0(2)$ \\
\hline $\mathrm{C} 1-\mathrm{C} 2-\mathrm{C} 3$ & $118.07(18)$ & $\mathrm{C} 2-\mathrm{C} 1-\mathrm{H} 1$ & 120.00 \\
\hline $\mathrm{N} 1-\mathrm{C} 3-\mathrm{C} 2$ & $118.53(18)$ & $\mathrm{C} 6-\mathrm{C} 1-\mathrm{H} 1$ & 120.00 \\
\hline $\mathrm{N} 1-\mathrm{C} 3-\mathrm{C} 4$ & $118.41(19)$ & $\mathrm{C} 1-\mathrm{C} 2-\mathrm{H} 2$ & 121.00 \\
\hline $\mathrm{C} 2-\mathrm{C} 3-\mathrm{C} 4$ & $123.1(2)$ & $\mathrm{C} 3-\mathrm{C} 2-\mathrm{H} 2$ & 121.00 \\
\hline $\mathrm{C} 3-\mathrm{C} 4-\mathrm{C} 5$ & $118.17(19)$ & $\mathrm{C} 3-\mathrm{C} 4-\mathrm{H} 4$ & 121.00 \\
\hline $\mathrm{C} 4-\mathrm{C} 5-\mathrm{C} 6$ & $120.72(19)$ & $\mathrm{C} 5-\mathrm{C} 4-\mathrm{H} 4$ & 121.00 \\
\hline $\mathrm{C} 1-\mathrm{C} 6-\mathrm{C} 5$ & $119.24(18)$ & $\mathrm{C} 4-\mathrm{C} 5-\mathrm{H} 5$ & 120.00 \\
\hline $\mathrm{C} 1-\mathrm{C} 6-\mathrm{C} 7$ & $118.47(16)$ & $\mathrm{C} 6-\mathrm{C} 5-\mathrm{H} 5$ & 120.00 \\
\hline $\mathrm{C} 5-\mathrm{C} 6-\mathrm{C} 7$ & $122.29(18)$ & $\mathrm{O} 1-\mathrm{C} 8-\mathrm{H} 8 \mathrm{~A}$ & 110.00 \\
\hline $\mathrm{O} 2-\mathrm{C} 7-\mathrm{C} 6$ & $122.01(18)$ & $\mathrm{O} 1-\mathrm{C} 8-\mathrm{H} 8 \mathrm{~B}$ & 110.00 \\
\hline $\mathrm{O} 2-\mathrm{C} 7-\mathrm{C} 8$ & $122.19(19)$ & $\mathrm{C} 7-\mathrm{C} 8-\mathrm{H} 8 \mathrm{~A}$ & 110.00 \\
\hline $\mathrm{C} 6-\mathrm{C} 7-\mathrm{C} 8$ & $115.80(16)$ & $\mathrm{C} 7-\mathrm{C} 8-\mathrm{H} 8 \mathrm{~B}$ & 110.00 \\
\hline $\mathrm{O} 1-\mathrm{C} 8-\mathrm{C} 7$ & $109.30(16)$ & $\mathrm{H} 8 \mathrm{~A}-\mathrm{C} 8-\mathrm{H} 8 \mathrm{~B}$ & 108.00 \\
\hline $\mathrm{O} 1-\mathrm{C} 9-\mathrm{O} 3$ & $121.9(2)$ & $\mathrm{C} 11-\mathrm{C} 12-\mathrm{H} 12$ & 120.00 \\
\hline $\mathrm{O} 1-\mathrm{C} 9-\mathrm{C} 10$ & $111.65(16)$ & $\mathrm{C} 13-\mathrm{C} 12-\mathrm{H} 12$ & 120.00 \\
\hline $\mathrm{O} 3-\mathrm{C} 9-\mathrm{C} 10$ & $126.36(19)$ & $\mathrm{C} 12-\mathrm{C} 13-\mathrm{H} 13$ & 120.00 \\
\hline $\mathrm{C} 9-\mathrm{C} 10-\mathrm{C} 11$ & $122.06(17)$ & $\mathrm{C} 14-\mathrm{C} 13-\mathrm{H} 13$ & 120.00 \\
\hline $\mathrm{C} 9-\mathrm{C} 10-\mathrm{C} 15$ & $120.13(18)$ & $\mathrm{C} 13-\mathrm{C} 14-\mathrm{H} 14$ & 120.00 \\
\hline $\mathrm{C} 11-\mathrm{C} 10-\mathrm{C} 15$ & $117.74(18)$ & $\mathrm{C} 15-\mathrm{C} 14-\mathrm{H} 14$ & 120.00 \\
\hline $\mathrm{C} 11-\mathrm{C} 11-\mathrm{C} 10$ & $122.61(16)$ & $\mathrm{C} 10-\mathrm{C} 15-\mathrm{H} 15$ & 120.00 \\
\hline $\mathrm{C} 11-\mathrm{C} 11-\mathrm{C} 12$ & $116.66(17)$ & $\mathrm{C} 14-\mathrm{C} 15-\mathrm{H} 15$ & 119.00 \\
\hline $\mathrm{C} 9-\mathrm{O} 1-\mathrm{C} 8-\mathrm{C} 7$ & $-164.95(16)$ & $\mathrm{C} 5-\mathrm{C} 6-\mathrm{C} 7-\mathrm{O} 2$ & $177.1(2)$ \\
\hline $\mathrm{C} 8-\mathrm{O} 1-\mathrm{C} 9-\mathrm{O} 3$ & $5.3(3)$ & $\mathrm{C} 5-\mathrm{C} 6-\mathrm{C} 7-\mathrm{C} 8$ & $-2.8(3)$ \\
\hline $\mathrm{C} 8-\mathrm{O} 1-\mathrm{C} 9-\mathrm{C} 10$ & $-177.15(15)$ & $\mathrm{O} 2-\mathrm{C} 7-\mathrm{C} 8-\mathrm{O} 1$ & $3.6(3)$ \\
\hline $\mathrm{O} 4-\mathrm{N} 1-\mathrm{C} 3-\mathrm{C} 2$ & $174.8(3)$ & $\mathrm{C} 6-\mathrm{C} 7-\mathrm{C} 8-\mathrm{O} 1$ & $-176.59(15)$ \\
\hline $\mathrm{O} 4-\mathrm{N} 1-\mathrm{C} 3-\mathrm{C} 4$ & $-5.1(4)$ & $\mathrm{O} 1-\mathrm{C} 9-\mathrm{C} 10-\mathrm{C} 11$ & $170.13(17)$ \\
\hline $\mathrm{O} 5-\mathrm{N} 1-\mathrm{C} 3-\mathrm{C} 2$ & $-5.4(3)$ & $\mathrm{O} 1-\mathrm{C} 9-\mathrm{C} 10-\mathrm{C} 15$ & $-13.2(3)$ \\
\hline $\mathrm{O} 5-\mathrm{N} 1-\mathrm{C} 3-\mathrm{C} 4$ & $174.7(3)$ & $\mathrm{O} 3-\mathrm{C} 9-\mathrm{C} 10-\mathrm{C} 11$ & $-12.5(3)$ \\
\hline $\mathrm{C} 6-\mathrm{C} 1-\mathrm{C} 2-\mathrm{C} 3$ & $-0.2(3)$ & $\mathrm{O} 3-\mathrm{C} 9-\mathrm{C} 10-\mathrm{C} 15$ & $164.3(2)$ \\
\hline $\mathrm{C} 2-\mathrm{C} 1-\mathrm{C} 6-\mathrm{C} 5$ & $0.4(3)$ & $\mathrm{C} 9-\mathrm{C} 10-\mathrm{C} 11-\mathrm{C} 11$ & $-6.6(3)$ \\
\hline $\mathrm{C} 2-\mathrm{C} 1-\mathrm{C} 6-\mathrm{C} 7$ & $-178.83(17)$ & $\mathrm{C} 9-\mathrm{C} 10-\mathrm{C} 11-\mathrm{C} 12$ & $175.2(2)$ \\
\hline $\mathrm{C} 1-\mathrm{C} 2-\mathrm{C} 3-\mathrm{N} 1$ & $179.79(19)$ & $\mathrm{C} 15-\mathrm{C} 10-\mathrm{C} 11-\mathrm{C} 11$ & $176.60(17)$ \\
\hline $\mathrm{C} 1-\mathrm{C} 2-\mathrm{C} 3-\mathrm{C} 4$ & $-0.4(3)$ & $\mathrm{C} 15-\mathrm{C} 10-\mathrm{C} 11-\mathrm{C} 12$ & $-1.6(3)$ \\
\hline $\mathrm{N} 1-\mathrm{C} 3-\mathrm{C} 4-\mathrm{C} 5$ & $-179.5(2)$ & $\mathrm{C} 9-\mathrm{C} 10-\mathrm{C} 15-\mathrm{C} 14$ & $-175.3(2)$ \\
\hline $\mathrm{C} 2-\mathrm{C} 3-\mathrm{C} 4-\mathrm{C} 5$ & $0.7(4)$ & $\mathrm{C} 11-\mathrm{C} 10-\mathrm{C} 15-\mathrm{C} 14$ & $1.6(3)$ \\
\hline $\mathrm{C} 3-\mathrm{C} 4-\mathrm{C} 5-\mathrm{C} 6$ & $-0.5(4)$ & $\mathrm{C} 11-\mathrm{C} 11-\mathrm{C} 12-\mathrm{C} 13$ & $-178.1(2)$ \\
\hline $\mathrm{C} 4-\mathrm{C} 5-\mathrm{C} 6-\mathrm{C} 1$ & $0.0(3)$ & $\mathrm{C} 10-\mathrm{C} 11-\mathrm{C} 12-\mathrm{C} 13$ & $0.2(3)$ \\
\hline $\mathrm{C} 4-\mathrm{C} 5-\mathrm{C} 6-\mathrm{C} 7$ & $179.2(2)$ & $\mathrm{C} 11-\mathrm{C} 12-\mathrm{C} 13-\mathrm{C} 14$ & $1.3(4)$ \\
\hline $\mathrm{C} 1-\mathrm{C} 6-\mathrm{C} 7-\mathrm{O} 2$ & $-3.7(3)$ & $\mathrm{C} 12-\mathrm{C} 13-\mathrm{C} 14-\mathrm{C} 15$ & $-1.3(4)$ \\
\hline $\mathrm{C} 1-\mathrm{C} 6-\mathrm{C} 7-\mathrm{C} 8$ & $176.42(17)$ & $\mathrm{C} 13-\mathrm{C} 14-\mathrm{C} 15-\mathrm{C} 10$ & $-0.2(4)$ \\
\hline
\end{tabular}


supporting information

Hydrogen-bond geometry $\left(\AA,{ }^{\circ}\right)$

\begin{tabular}{lllll}
\hline$D-\mathrm{H} \cdots A$ & $D-\mathrm{H}$ & $\mathrm{H} \cdots A$ & $D \cdots A$ & $D-\mathrm{H} \cdots A$ \\
\hline $\mathrm{C} 2-\mathrm{H} 2 \cdots \mathrm{O}^{\mathrm{i}}$ & 0.93 & 2.54 & $3.258(2)$ & 135 \\
$\mathrm{C} 8-\mathrm{H} 8 A \cdots \mathrm{O} 3^{\mathrm{ii}}$ & 0.97 & 2.59 & $3.553(3)$ & 171 \\
$\mathrm{C} 13-\mathrm{H} 13 \cdots \mathrm{Cl} 1^{\mathrm{iii}}$ & 0.93 & 2.82 & $3.670(3)$ & 153 \\
$\mathrm{C} 14-\mathrm{H} 14 \cdots \mathrm{O} 44^{\mathrm{i}}$ & 0.93 & 2.50 & $3.211(4)$ & 134
\end{tabular}

Symmetry codes: (i) $-x+1, y+1 / 2,-z+1 / 2$; (ii) $x,-y+1 / 2, z-1 / 2$; (iii) $-x, y+1 / 2,-z+3 / 2$. 